\title{
A Survey on Techniques for Brain Tumor Segmentation from Mri
}

\author{
Harsimranjot Kaur ${ }^{1}$, Dr. Reecha Sharma ${ }^{2}$ \\ ${ }^{I}$ (M. Tech Student, Department of ECE, Punjabi University, Patiala, India) \\ ${ }^{2}$ (Assistant Professor, Department of ECE, Punjabi University, Patiala, India)
}

\begin{abstract}
Biomedical images are the core of medical science. Magnetic Resonance Imaging (MRI) scans produce detailed images of the internal structure of human brain and other parts of the body. Brain tumor segmentation from brain MRI data is a very significant task but it is done manually by medical experts which is very cumbersome and time-consuming. A large number of techniques have been proposed for the automatic brain tumor detection and segmentation from the brain MRI images. In this paper, the existing brain tumor detection and segmentation techniques for brain MRI images have been discussed.
\end{abstract}

Keywords: Brain MRI images, FCM, Region growing, Thresholding, Tumor segmentation.

\section{Introduction}

In the field of biomedical imaging, the segmentation of tumor from the human brain has become an emergent area of research and hence diverse literature is available in the field. For the diagnosis and treatment of the patient suffering from brain tumor, doctors take the help of MRI scans of the brain. But the diagnosis of the MRI scan is done manually by the doctor which is a time consuming task and the accuracy of the result depends on the experience of the doctor. The conclusions may vary from one doctor to another. Therefore, there is a need to overcome these problems and to automate the analytical process of brain tumor detection in MRI images. For this, biomedical image processing techniques are applied on the MRI scans [1] [2]. Hence, the segmentation and further classification of brain tumor from the MRI scans remains an extensive area of research in the field of medical science. In this paper, various existing techniques for detection and segmentation of brain tumor from MRI images i.e. thresholding-based, edge-based, region-based and clustering-based segmentation have been discussed.

\section{Related Work}

In the literature, there are a large number of existing techniques and algorithms for the detection and segmentation of brain tumor from MRI images. Asra Aslam et al. presented an improved edge detection algorithm for brain tumor segmentation [3]. This algorithm combines the Sobel method with image dependent thresholding, finds closed regions using closed contour algorithm and extracts tumors from the image. The brain tumors extracted by proposed algorithm are better than the tumors extracted using Sobel edge detector, Roberts edge detector and Prewitt edge detector. Roy et al. proposed a completely automatic algorithm to identify tumors by utilizing symmetry analysis [4]. In this paper, the idea that the region of image containing the tumor has higher intensity than the region with healthy brain tissues is being used. The MRI image is enhanced, filtered and segmentation is done and it also produces great results in the images having non-uniform contrast distributions. Kharrat et al. proposed an algorithm for detection of brain tumor from MRI images [5]. In this paper, the morphological operations, wavelet decomposition and k-means algorithm for segmentation is implemented to extract tumor area. The results show that the algorithm is feasible and performs very well on MRI images. S. Roy et al. explored a technique to distinguish tumor in brain MRI [6]. In this paper, image enhancement, morphological operations and watershed segmentation are applied. Results demonstrate that Watershed Segmentation can effectively extract a tumor if the parameters are set properly before segmentation. Malakooti et al. proposed a tumor segmentation technique which combines both fuzzy logic and neural networks and extracts the boundary taking into account level set method [7]. The proposed technique gives better results as compared to other existing techniques. M. K. Behera et al. proposed a novel fast and robust fuzzy c-means clustering framework for image segmentation based on local spatial and gray information [8]. This method has low computational time, less complexity and the algorithm is effective and efficient. Cai et al. proposed a fuzzy clustering algorithm that utilizes dependable neighbor pixels for image segmentation [9]. The proposed algorithm uses the local statistical data to separate dependable neighbor pixels thereby improving the segmentation performance and the result of segmentation is adaptive to the original image. Gopinath et al. portrayed the proposed system for recognition and extraction of prostate cancer cells from the MRI image of the prostate organ [10]. In this paper, noise removal from MRI image by high pass and median filtering and then segmentation of MRI image is done by threshold segmentation, watershed segmentation and morphological 
operations. The extracted regions of cancerous cell from the input MRI image are extracted efficiently. R. B. Dubey et al. proposed a semi-automated region growing segmentation algorithm for the brain tumor region segmentation from the MRI images [11]. This proposed segmentation method extracts tumor region efficiently if the parameters are set properly. The techniques discussed in the above literature review have been tabulated as given below:

Table.1: Several techniques for brain tumor segmentation from MRI images

\begin{tabular}{|l|l|l|}
\hline S.No. & Author & Technique Used For Brain Tumor Segmentation \\
\hline 1. & Asra Aslam et al. & Image dependent thresholding method combined with Sobel operator \\
\hline 2. & Roy et al. & Automatic algorithm for segmentation of brain tumor from MRI images \\
\hline 3. & Kharrat et al. & Wavelet based decomposition and k-means algorithm \\
\hline 4. & S. Roy et al. & $\begin{array}{l}\text { Tumor extraction from MRI image following morphological operators and watershed } \\
\text { segmentation }\end{array}$ \\
\hline 5. & Malakooti et al. & Segmentation combining both fuzzy logic and neural networks \\
\hline 6. & M. K. Behera et al. & Robust fcm clustering framework \\
\hline 7. & Cai et al. & Fuzzy clustering algorithm using dependable neighbor pixels \\
\hline 8. & Gopinath et al. & Threshold segmentation and morphological operators with watershed segmentation \\
\hline 9. & R. B. Dubey et al. & Semi-automated region growing segmentation method \\
\hline
\end{tabular}

\section{Existing Mri Brain Image Segmentation Techniques}

The existing techniques for segmentation of brain tumor from MRI images can be broadly classified into four categories, viz. threshold based segmentation, edge based segmentation, region based segmentation and clustering based segmentation techniques. In threshold based segmentation techniques, the objects from the image are extracted on the basis of a particular threshold. In edge based segmentation techniques, the abrupt change in intensity values is taken into consideration for object extraction. In region based segmentation techniques, the image is divided into regions having different properties. In the case of clustering based segmentation techniques, an image is divided into a number of clusters based on the value of membership functions allotted to each pixel in the image. Fig. 1 represents the classification of these segmentation techniques.

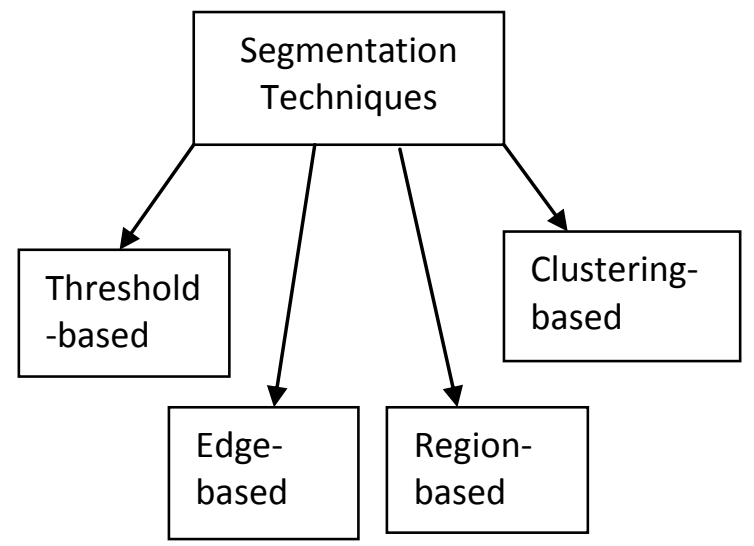

Fig.1: Classification of segmentation techniques on the basis of pixel intensity.

\subsection{Thresholding}

Thresholding is one of the frequently used methods for image segmentation [12]. This method is suitable for images with different intensities of pixels. Using this method, the image is partitioned directly into different regions based on these intensity values of the pixels.

\section{(A) Global thresholding}

Global thresholding method chooses only one threshold value for the entire image. Global thresholding is used for bimodal images. It is simpler and faster in computational time only if the image has uniform intensity distribution and high contrast between foreground and background.

- Otsu's thresholding

Otsu's thresholding method depends on a discriminant analysis which divides the image into two classes based on the intensity of gray levels in the image [13]. The main advantage of Otsu's method is that it is simple and effective to implement. While Otsu's method can segment only larger objects from background and fails, if the image has variable contrast distribution. 


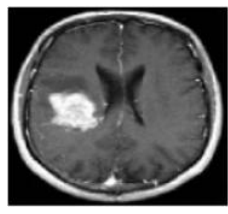

(a)

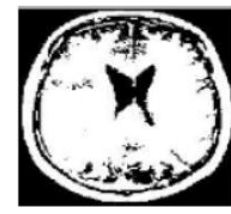

(b)

Fig.2: Otsu's thresholding method: (a) original input image; (b) Output of Otsu's method.

\section{(B) Local thresholding}

Threshold values are chosen locally by dividing an image into sub-images and threshold value for each part is calculated. A local thresholding technique takes more computational time than the global thresholding. Its result is satisfactory in an image with background variations. It can extract only smaller regions.

- Histogram thresholding

Histogram thresholding segmentation is based upon the thresholding of histogram features and gray-level thresholding in an image. It gives better results but the computational time is more for histogram thresholding.

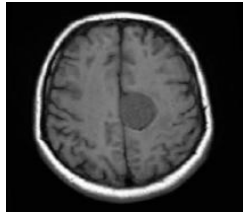

(a)

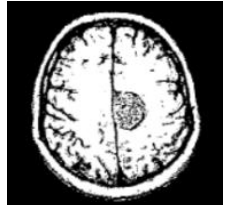

(b)

Fig.3: Histogram thresholding method: (a) original input image; (b) Output of histogram thresholding.

\subsection{Edge Based Segmentation}

Edge based segmentation methods divide an image based on abrupt changes in the intensity of pixels near the edges [14]. The result is a binary image with edges of the objects being detected. Based on the theory, there are two basic edge based segmentation methods viz. gray histogram and gradient based methods.

\section{(A) Gray Histogram Technique}

The result of the technique of gray histogram mainly depends upon selection of threshold $(\mathrm{T})$. The image is converted into grayscale image and after that gray-level thresholding is applied on the histogram of that image.

\section{(B) Gradient Based Method}

In the gradient based method, the difference between intensity values of neighboring pixels is taken into account [15]. So, when there is an abrupt change in the intensity in a region of an image and there is very less image noise then gradient based methods works well. These methods involve applying gradient operators on the image. The basic edge detection operators used in this method are Sobel operator, Canny operator, Laplace operator, Laplacian of Gaussian (LOG) operator etc., out of which Sobel and Canny operators produce better results Edge detection methods exhibit a balance between accuracy and noise immunity. If the level of detecting accurate edges is too high, then noise may produce fake edges and if the degree of noise immunity is too high, then some parts of the image containing important information might go undetected.

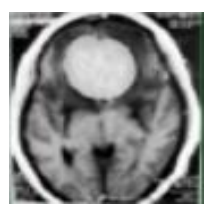

(a)

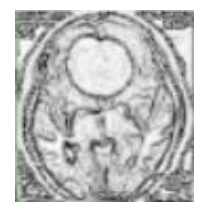

(b)

Fig.4: Edge based method: (a) original input image; (b) Output of Sobel operator.

\subsection{Region Based Segmentation}

Region based methods divide an image into regions that are similar on the basis of a set of a particular criterion [16]. The existing region segmentation techniques mainly consist of the following methods:

\section{(A) Region growing}

Region growing method is one of the most frequently used segmentation methods. This method initiates with a seed pixel and grows the region by incorporating the neighboring pixels based on some threshold 
value [17]. The region growing stops when all pixels belong to some particular regions. Region growing segmentation is particularly used for small and simple structures such as tumors, lesions etc.

\section{(B) Region splitting and merging}

The image is split into a number of different regions depending on some criterion and after the splitting, it is merged. The whole image is initially considered as a single region and then the internal similarity of the image is calculated using standard deviation. If the variation is very large, then the image is split into regions using some threshold value. This process is repeated until no more further splitting of the region is possible. Quad tree is a common data structure used for splitting.

\section{(C) Watershed segmentation}

Watershed segmentation algorithm can be used if the image has uniform contrast distribution and the intensity of the foreground and background is distinguishable. Watershed algorithm is also used to find the weak edges in the images.

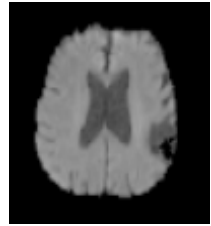

(a)

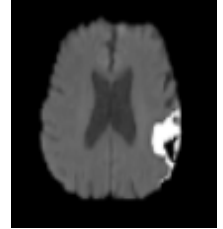

(b)

Fig.5: Region-based segmentation: (a) original input image; (b) Output of region growing.

\subsection{Clustering}

Clustering is the technique which is most frequently used in the MRI Segmentation, where it divides pixels into classes, without having prior information or training [18]. It classifies the pixels having largest probability into the same class. In the clustering technique, the training is done by utilizing the pixel characteristics with properties of each class of classified pixels.

\section{(A) K-means}

K-means clustering algorithm is the simplest of the existing clustering algorithms that can do clustering of pixels into numerous regions based on pixel properties. This method is called hard clustering as the clusters must be distant enough from each other and every pixel is assigned the membership function in such a way that it belongs to one particular region only.

\section{(B) Fuzzy C-means (FCM)}

FCM clustering is an unsupervised method for the analysis of given input image [19]. The fcm clustering algorithm assigns membership functions to every pixel in an image corresponding to each cluster center based on the distance of the cluster center from that particular pixel. The pixels near to the cluster center have higher membership function towards the particular cluster center.

\section{(C) Hierarchical clustering}

Hierarchical clustering method works by grouping data objects in an image into a tree of clusters. Hierarchical clustering does not need to specify the number of clusters in advance.

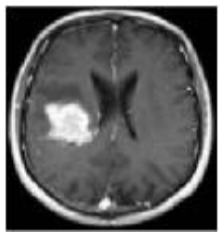

(a)

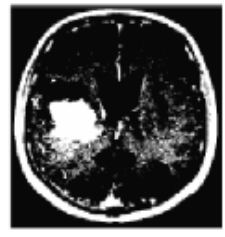

(b)

Fig.6: Clustering-based segmentation: (a) original input image; (b) Output of fuzzy c-means clustering.

\section{Summary And Conclusions}

In this paper, several existing brain tumor detection and segmentation techniques for brain MRI images have been discussed. The various existing segmentation techniques like thresholding-based, region-based, edgebased and clustering-based segmentation techniques have been described for the extraction of brain tumor from MRI images. The intensity-based thresholding methods provide good results but fail for the images with large intensity differences. The region-based segmentation is good for high contrast images but for low contrast 
images, it does not provide efficient results. Edge-based segmentation provides better results but fail for noisy images as it produces false edges for them. Clustering-based segmentation is very simple, fast and provides good results but for noisy images, it produces inaccurate results.

\section{Future Scope}

From the literature survey, it has been found that there is no universal system that can detect the tumor accurately regardless of its location, shape and intensity. Therefore, this topic further can further be explored, so that a better tumor detection system can be built which can help the doctors in evaluating MRI scans as the automated system will take lesser time than manual analysis and will provide more accurate results which will eventually be helpful in the treatment of patients suffering from brain tumor.

\section{References}

[1]. Gordillo, Nelly, Eduard Montseny, and Pilar Sobrevilla, "State of the art survey on MRI brain tumor segmentation", Magnetic resonance imaging, Elsevier, vol. 31, no. 8, pp. 1426-1438, 2013.

[2]. Aswathy, S. U., G. Glan Deva Dhas, and S. S. Kumar, "A survey on detection of brain tumor from MRI brain images", In Control, Instrumentation, Communication and Computational Technologies (ICCICCT), International Conference on, pp. 871-877, IEEE, 2014.

[3]. Aslam, Asra, Ekram Khan, and MM Sufyan Beg, "Improved Edge Detection Algorithm for Brain Tumor Segmentation", Procedia Computer Science, vol. 58, pp. 430-437, 2015.

[4]. Roy, Sudipta, and Samir K. Bandyopadhyay, "Detection and Quantification of Brain Tumor from MRI of Brain and its Symmetric Analysis”, International Journal of Information and Communication Technology Research, vol. 2, no. 6, 2012.

[5]. Kharrat Ahmed et al. "Detection of brain tumor in medical images", In Signals, Circuits and Systems (SCS), 3rd International Conference on IEEE, pp. 1-6, Nov 2009.

[6]. Roy, Sudipta et al. "Brain Tumor segmentation and quantification from MRI of brain", Journal of Global Research in Computer Science, vol. 2, no. 4, pp. 155-159, 2011

[7]. M.V. Malakooti, S. A. Mousavi and N. H. Taba, "MRI brain Image Segmentation Using Combined Fuzzy Logic and Neural Networks for the Tumor Detection", Journal of Academic and Applied Studies, vol. 3, no. 5, 2013.

[8]. Manoj Kumar Behera et al. "An approach for image segmentation using fuzzy c-means clustering", International Journal of Multidisciplinary Research and Development, vol. 2, no. 6, pp. 349-4182, 2015.

[9]. W.Cai, S.Chen and L.Lei, “A Fuzzy Clustering Algorithm for Image Segmentation using Dependable Neighbor Pixels”, In Pattern Recognition, Chinese Conference on, pp. 1-5, IEEE, 2009.

[10]. Gopinath N. et al. "Extraction of Cancer Cells from MRI Prostate Images using matlab", International Journal of Engineering Science and Innovative Technology (IJESIT), vol. 1, 2012.

[11]. Dubey, R. B., M. Hanmandlu, and S. K. Gupta, "Region growing for MRI brain tumor volume analysis", Indian Journal of Science and Technology, vol. 2, no. 9, pp. 26-31, 2009.

[12]. Rajeshwar Dass, Priyanka, Swapna Devi, "Image Segmentation Techniques", International Journal of Electronics \& Communication Technology (IJECT), vol. 3, no. 1, pp. 2230-7109, 2012.

[13]. D. Selvaraj et al. "MRI Brain Image Segmentation Techniques - A Review", Indian Journal of Computer Science and Engineering (IJCSE), vol. 4, no. 5, pp. 0976-5166, 2013.

[14]. G. Evelin Sujji, Y.V.S. Lakshmi, G. Wiselin Jiji, "MRI Brain Image Segmentation based on Thresholding", International Journal of Advanced Computer Research, vol. 3, no. 1, issue 8, pp. 2249-7277, March 2013.

[15]. Aswathy, S. U., G. Glan Deva Dhas, and S. S. Kumar, "A survey on detection of brain tumor from MRI brain images", In Control, Instrumentation, Communication and Computational Technologies (ICCICCT), International Conference on, pp. 871-877, IEEE, 2014.

[16]. Rohan Kandwal et al. "Review: Existing Image Segmentation Techniques", International Journal of Advanced Research in Computer Science and Software Engineering, vol. 4, no. 4, pp. 2277-128X, 2014.

[17]. Viji, K.A. and JayaKumari, J., "Modified texture based region growing segmentation of MR brain images", In Information \& Communication Technologies (ICT), IEEE Conference on, pp. 691-695, IEEE, April 2013.

[18]. Shen, Shan, William Sandham, Malcolm Granat, and Annette Sterr, "MRI fuzzy segmentation of brain tissue using neighborhood attraction with neural-network optimization", IEEE transactions on information technology in biomedicine, vol. 9, no. 3, pp. 459467, 2005.

[19]. Ahmed, M.N., Yamany, S.M., Mohamed, N., Farag, A.A. and Moriarty, T., “A modified fuzzy c-means algorithm for bias field estimation and segmentation of MRI data", IEEE transactions on medical imaging, vol. 21, no. 3, pp.193-199, 2002. 